\title{
X-ray Emission from Dwarf Galaxies: IC 2574 Revisited
}

\author{
Elias Brinks (ebrinks@inaoep.mx)* \\ INAOE, Apdo. Postal 51 \& 216, Puebla, Pue 72000, Mexico \\ Fabian Walter (fwalter@nrao.edu) \\ NRAO, AOC, P.O. Box O, Socorro, NM 87801, USA \\ Jürgen Kerp (jkerp@astro.uni-bonn.de) \\ RAIUB, Auf dem Hügel 71, 53121 Bonn, Gerany
}

September 30, 2002

\begin{abstract}
Until recently, few unequivocal detections had been reported of the hot, $\mathrm{X}$-ray emitting gas thought to be associated with the large, coherent structures variously described as supershells or superbubbles in dwarf irregular (dIrr) galaxies. In this contribution we report follow-up XMM-Newton and Chandra observations of our ROSAT detection of $\mathrm{X}$-ray emission associated with the supergiant shell in the nearby dIrr galaxy IC 2574, a member of the M 81 group of galaxies. The spectral properties of the X-ray source suggest that we are dealing with a young (age $<2000 \mathrm{yr}$ ) supernova remnant (SNR). This SNR is most likely one of the many supernovae which have exploded in that region and which have created the impressive supergiant $\mathrm{HI}$ shell.
\end{abstract}

Keywords: galaxies: individual (IC 2574), galaxies: ISM, X-rays

\section{Introduction}

The interstellar medium (ISM) in gas rich spiral and dwarf irregular (dIrr) galaxies is in pressure equilibrium, the energy input being provided by supernova explosions. As star formation tends to occur in groups and associations, this implies that Type II supernovae (SNe) will explode within a relatively short time span and within a confined volume. The accumulated energy of several tens to hundreds of SNe heat up this volume to coronal temperatures and the overpressure will cause it to expand, creating coronal gas filled cavities within the ISM of gas rich galaxies. The features thus created are known as (super)bubbles or (super)giant shells and can reach dimensions of up to a kiloparsec. These shells leave their mark on the surrounding neutral ISM which when mapped in the 21-cm line of neutral hydrogen (HI) takes on the appearance of a swiss cheese.

Naively one might expect the coronal gas to be easily detectable through its thermal $\mathrm{X}$-ray emission. However, detecting this hot plasma has been trickier than thought at first. It has only been thanks to the

\footnotetext{
* on sabbatical leave from the Universidad de Guanajuato, Guanajuato, Mexico
} (C) 2007 Kluwer Academic Publishers. Printed in the Netherlands. 

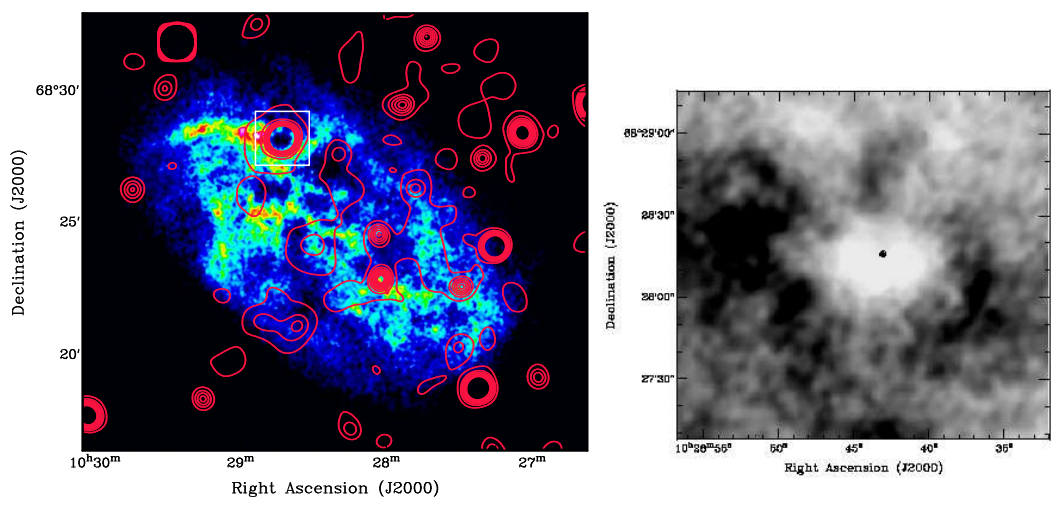

Figure 1. Left: X-ray observations of IC 2574 obtained with XMM-Newton overlaid as contours on a greyscale representation of the HI column density map from Walter \& Brinks (1999). The XMM-Newton map resultsed from a preliminary calibration and reduction of data over a $0.5-4 \mathrm{keV}$ range in energy. Right: Blow-up of the area indicated by the white square in the image on the left, showing the HI data of the region corresponding to the supergiant shell as a grey scale representation (white implying low HI column density) with overlaid as contours our Chandra observation. (Kerp et al., in preparation).

improved sensitivity of ROSAT compared to earlier X-ray missions that the hot $\mathrm{X}$-ray emitting plasma from supergiant shells in the Magellanic Clouds could be mapped (Points et al., 2000; Dunne et al., 2001). Given typical luminosities of $10^{35}$ to $5 \times 10^{36} \mathrm{erg} \mathrm{s}^{-1}$, galaxies further afield remained beyond the reach of ROSAT and had to await the launch of Chandra and XMM-Newton.

At this point we should like to make a distinction between the detection of coronal gas confined within the ISM of galaxies and the extended emission seen in far more violent environments such as in starburst galaxies (a galaxy is considered as undergoing a starburst if the current star formation rate would exhaust the supply of "fuel" in much less than a Hubble time). ROSAT did detect outflows of coronal gas from starburst galaxies, out to respectable distances and even from dwarf galaxies undergoing a starburst, such as NGC 1569 (Heckman et al., 1995), NGC 3077 (Bi et al., 1994), NGC 4449 (Vogler \& Pietsch, 1997) and NGC 5253 (Martin \& Kennicutt, 1995; Strickland \& Stevens, 1999). Follow-up observations of starburst dwarf galaxies with Chandra have been presented by Martin et al. (2002), Ott et al. (2002), and Ott (2002). In this contribution, though, we focus on dwarf galaxies which are not classified as being a starburst.

One of the best candidates outside of the Local Group to search for X-ray emission from a supergiant shell is IC 2574. This dIrr galaxy is part of the M 81 group, located at a distance of 3.6 Mpc. Towards 
the northeast we find a region of active star formation, not unlike the 30 Dor region in the LMC. This region, known as IC 2574-SGS (for supergiant shell) has an $\mathrm{H} \alpha$ luminosity of about $30 \%$ of 30 Dor. The $\mathrm{H} \alpha$ emission forms a ring which coincides with an expanding ring of neutral hydrogen as seen in HI (Walter \& Brinks, 1999).

\section{X-ray Observations of IC 2574}

A $7.3 \mathrm{ksec}$ ROSAT exposure revealed what appeared to be a marginally resolved source filling the HI cavity (Walter et al., 1998). In Figure 1 we show follow-up observations with the XMM-Newton $(20 \mathrm{ksec})$ and Chandra (10 ksec) X-ray observatories. The former has superior sensitivity at modest, 15", angular resolution whereas the latter, at $1^{\prime \prime}$, approaches resolutions similar to that of optical images. The left-hand panel shows as contours a prelinimary map of the XMM-Newton results superposed on an HI image of the galaxy. In addition to several unresolved sources, most of them unrelated to the galaxy, and some suggestion for extended emission which coincides with features in the HI map, a strong source is detected at the position of the IC 2574-SGS. The sensitivity limit at the distance of $3.6 \mathrm{Mpc}$ of the $X M M-N e w t o n$ map is about $5 \times 10^{36} \mathrm{erg} \mathrm{s}^{-1}$, or comparable to the brighter superbubbles detected in the LMC. The right-hand panel shows a blow-up of the SGS with superposed the higher resolution Chandra data, again as contours.

\section{Results and Discussion}

Contrary to what we thought on the basis of the ROSAT observations, the new Chandra data show that the source at the center of the SGS in IC 2574 is unresolved, hence we are not seeing the extended X-ray emission from the hot gas which is supposedly filling the SGS. An upper limit for the linear size is $\sim 8$ pc. In Figure 2 we show the spectrum of this source, observed with Chandra. Spectral fitting of the data, adopting $20 \%$ solar metallicity for the gas in IC 2574, and assuming a foreground absorbing column density (at solar metallicity) of $N_{\mathrm{HI}}=8 \times 10^{20} \mathrm{~cm}^{-2}$ suggests a two-component model, consisting of a thermal plasma model (Mewe-Kaastra) and a power law component. The thermal plasma model leads to a temperature of $\mathrm{kT}=2.4 \mathrm{keV}$ and dominates the observed spectrum at the low energy end. At the high energy side the spectrum is well represented by a power law component with a power law index of $1.0 \pm 0.2$ (Kerp et al., in preparation). 


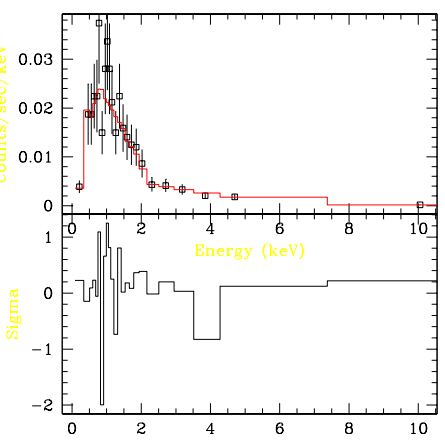

Figure 2. Top: X-ray spectrum as obtained with Chandra towards the unresolved source IC 2574-SGS. The solid line represents a fit to the spectrum using a hybrid model consisting of thermal plasma emission and a power law component (see text). bottom: residuals of the model fit.

The power law component, if originating from a source in IC 2574 has a luminosity close to the Eddington limit (it is overluminous by perhaps $10 \%$ ) and could be attributed to a cooling neutron star. The luminosity and spectral characteristics of the thermal plasma $(\mathrm{T}=$ $10^{7.4} \mathrm{~K}$ ) match that of a young SNR with an estimated age $<2000 \mathrm{yr}$. This SNR is likely one of the many supernovae which have gone off within the volume of the SGS and within the past $1.4 \times 10^{6} \mathrm{yr}$, which have created the impressive expanding HI shell.

EB gratefully acknowledges support from CONACyT via grant 27606E. JK likes to thank the Deutsches Zentrum für Luft- und Raumfahrt for finiancial support under grant No. 50 OR 0103.

\section{References}

Bi, H. G., Arp. H., \& Zimmermann, H. U. 1994, A\&A, 282, 386

Dunne, B. C., Points, S. D., \& Chu, Y.-H. 2001, ApJS, 136, 119

Heckman, T. M., Dahlem. M., Lehnert, M. D., Fabbiano, G., Gilmore, D., \& Waller, W. H. 1995, ApJ, 448, 98

Martin, C. L., \& Kennicutt, R. C., Jr. 1995, ApJ, 447, 171

Martin, C. L., Kobulnicky, H. A., \& Heckman, T. M. 2002, ApJ, 574, 663

Ott, J. 2002, PhD Thesis, Bonn University

Ott, J., Martin, C. L., \& Walter, F. 2002, ApJ (submitted)

Points, S. D., Chu, Y.-H., Snowden, S. L., \& Staveley-Smith, L. 2000, ApJ, 545, 827

Strickland, D. K., \& Stevens, I. R. 1999, MNRAS, 306, 43

Vogler, A., \& Pietsch, W. 1997, A\&A, 319, 459

Walter, F., Kerp, J., Duric, N., Brinks, E., \& Klein, U. 1998, ApJ, 502, L143

Walter, F, \& Brinks, E. 1999, AJ, 118, 273 